Children, Women and Children's Division, Oslo University Hospital, Oslo, Norway; ${ }^{5} T h e$ Nordic School of Public Health; ${ }^{6}$ Bracke Diakoni, Gothenburg, Sweden

Background and Aims Functional disability and bodily pain seem to be the most important determinants of physical and psychosocial well-being in young people with juvenile idiopathic arthritis (JIA) into adulthood. Disease course and self-reported daily life experiences through adolescence, and health professionals' guidance and counselling in the transition process to adult life are explored.

Method Telephone interviews based on a questionnaire including open-ended and closed questions among 51 informants as part of a second follow-up of a Norwegian cohort of patients with JIA 18.4 years after symptom onset. Descriptive statistics were used to analyse quantitative data. Data from the open-ended questions were categorised and quantified manually.

Results Main findings are the high frequency of symptoms related to JIA the previous year (70.6\%) and disease residua (78.4\%). A considerable proportion experienced limitations in education planning and job accessibility, yet 46 informants $(90.2 \%)$ felt satisfactory in their study and/or job situation. More than $55 \%$ of the informants answered that they to some/great extent were informed about illness related issues. However, more than 70\% answered 'no' when asked whether they had received counselling with regard to psychosocially related issues, and $70.6 \%$ stated that they had not been prepared for transfer to adult ward.

Conclusion JIA-related plagues and worries seem to impact the entire period of adolescence into adult life for a considerable proportion of the informants. Guidance and counselling should be formalised and should focus on both illness- and psychosocially related implications of the disease in young people with JIA.

\section{THE EFFECT OF HEAVY SCHOOL BAG WEIGHT ON PHYSICAL PROBLEMS IN CHILDREN AND ADOLESCENCE. SHIRAZ, IRAN}

doi:10.1136/archdischild-2012-302724.1892

N Beheshtipour, I Jahanbin, A Haghnegahdar. Pediatric, Shiraz University, Shiraz, Iran

Background One of the main physical problems that have been considered less is the physical complaints related to school bag weight that has a great adverse effect on the incidence of movement disorders in future.

Objective Evaluation of physical problems as consequences of over weight bag school.

Method and materials In this descriptive study, 1255 primary and guidance school students were selected from 16 schools randomly from four different regions of Shiraz. Demographic data were collected by a questionnaire. The data related to physical problems including the type of the bags, the type of transportation to school and the weight of students and their bags which were measured by a digital seca scale with the sensitivity of 100 grams. Data were analyzed by SPSS on way ANOVA, Chi-square and T-test.

Result Complaints from shoulder pain were the main problem $(41.2 \%)$. The mean of school bag weight was $2.81 \pm 1.09 \mathrm{~kg}$ that had significant correlation with the incidence of physical complaints $(\mathrm{P}=0.001)$ in all students. The school bag weight of 440 students (35.1\%) were over $10 \%$ of the student's body weight and $94 \%$ of them suffering from physical complaints.

Conclusion This study revealed strong correlation between school bag weight and physical problems among all students, and can lead to serious consequences in the future.

\section{SEXUAL ABUSED IN CHILD}

doi:10.1136/archdischild-2012-302724.1893

${ }^{1}$ A Nematollahi, ${ }^{2} Z$ Montaseri, ${ }^{2} Z$ Yazdanpanahi. ${ }^{1}$ Nursing; ${ }^{2}$ Nursing and Midwifery of Medical Science, Shiraz, Iran
Introduction Prevalence estimates suggest that between 12\% and $35 \%$ of women and between $4 \%$ and $9 \%$ of men report having experienced sexual abuse before 18 years of age. Although no sexual abuse-specific syndrome has been described, aggressive behaviour, social isolation, somatization, anxiety, depression, nightmares, inappropriate sexualized behaviours and symptoms of post-traumatic stress disorder (PTSD) are the most frequent sequelae reported.

Methods The following sources were searched: Medline and hand searches of relevant journals from 1998-2009.

Results The sequelae of child sexual abused indicates that children not only express short-term adaptation problems, but are also at risk of adolescence and adulthood revictimization, drug and alcohol abuse and a host of other symptoms. Vulnerability to victimization and varied outcomes are determined by interactions of three sets of mutually influential factors: personal variables, event factors, and environmental factors. Based on the literature, we predicted a high prevalence of psychiatric disturbance in these abused children $30 \%$ or greater). Children who have been both physically/sexually abused appear to be at highest risk of psychiatric disturbance. Results show that in the sexual abuse group, single-parent families were more frequent $(53.7 \%$ versus $32.3 \%$; $\mathrm{P}<0.01)$, mothers were less educated $(10.8 \%$ versus $13.1 \% ; \mathrm{P}<0.0001)$ and socioeconomic level was lower $(36.8 \%$ versus $47.9 \%$; $\mathrm{P}<0.0001)$.

Conclusions Child sexual abuse is a common problem in our society and medical professionals who provide evaluations of children who may have been abused need.

\section{JOB SATISFACTION SURVEY AMIONG PEDIATRIC ONCOLOGY NURSES}

doi:10.1136/archdischild-2012-302724.1894

${ }^{1} \mathrm{M}$ Soltanian, ${ }^{2} \mathrm{~F}$ Nikouee, ' $\mathrm{S}$ Montaseri, ${ }^{1} \mathrm{M}$ Edraki. ${ }^{1}$ Fatemeh College of Nursing 8 Midwifery, Shiraz University of Medical Sciences, Shiraz; ${ }^{2}$ Fasa University of Medical Science, Fasa, Iran

Purpose job satisfaction becoming an increasingly large disorder and defined as the degree to which employees enjoy their job. Job satisfaction is a significant predictor of nursing absenteeism, burnout, turnover and intention to quit; however, there have been some inconsistent findings. Much research has revealed that job satisfaction of pediatric oncology nurses is closely related to job stress, role conflict and ambiguity, organizational and professional commitment. This led the researcher to carry out a study to identify variables of job satisfaction among nurses working in pediatric oncology ward.

Method A descriptive cross sectional design was carried out by using quantitative data obtained through self-administered questionnaires. Our tool was the 'job satisfaction survey' to examine job satisfaction among 25 pediatric oncology nurses from SHIRAZ University of Medical Science (IRAN).

Results The job satisfaction for pediatric oncology staff nurses had been calculated using mean and standard deviation and results revealed that (N:25, Mean:2.3)and this is an indication of dissatisfaction

Conclusion Studies over the past decade have increasingly focused on job dissatisfaction placed on health professionals and the negative consequences of that. The implication for this study is that nursing work in pediatric oncology ward is a difficult profession and additional researches are needed to identifying positive strategies for reducing job dissatisfaction. this study indicates the importance of adopting strategies to demonstrate more social support for the pediatric oncology nurses in the workplace. EMERGENCY MEDICAL SYSTEM

doi:10.1136/archdischild-2012-302724.1895 